Wilfrid Laurier University

Scholars Commons @ Laurier

Physics and Computer Science Faculty

Publications

Physics and Computer Science

1987

\title{
Chaotic Behavior in an Array of Coupled Josephson Weak Links
}

M.A.H. Nerenberg

University of Western Ontario

J.H. Baskey

University of Western Ontario

James A. Blackburn

Wilfrid Laurier University, jabjabjab@cogeco.ca

Follow this and additional works at: https://scholars.wlu.ca/phys_faculty

\section{Recommended Citation}

Nerenberg, M.A.H.; Baskey, J.H.; and Blackburn, James A., "Chaotic Behavior in an Array of Coupled Josephson Weak Links" (1987). Physics and Computer Science Faculty Publications. 26.

https://scholars.wlu.ca/phys_faculty/26

This Article is brought to you for free and open access by the Physics and Computer Science at Scholars Commons @ Laurier. It has been accepted for inclusion in Physics and Computer Science Faculty Publications by an authorized administrator of Scholars Commons @ Laurier. For more information, please contact scholarscommons@wlu.ca. 


\title{
Chaotic behavior in an array of coupled Josephson weak links
}

\author{
M. A. H. Nerenberg and J. H. Baskey \\ Department of Applied Mathematics, University of Western Ontario, London, Ontario, Canada N6A SB9
}

James A. Blackburn

Physics Department, Wilfrid Laurier University, Waterloo, Ontario, Canada N2L 3C5

(Received 21 July 1987)

\begin{abstract}
Using the resistively shunted junction model, an array of three resistively coupled, noncapacitive, Josephson weak links, driven only by dc bias currents, is studied for the existence of chaos. This system represents one of the set of three simplest Josephson systems that, in principle, can have chaotic dynamics. The results attest to the validity of the Ruelle-Takens-Newhouse scenario for the onset of chaos.
\end{abstract}

\section{INTRODUCTION}

The resistively shunted junction (RSJ) model is generally accepted as successfully describing the behavior of various superconducting systems falling under the rubric of Josephson junction, including weak links. ${ }^{1}$ The corresponding equations are highly nonlinear and imply strong coherent ${ }^{2,3}$ and chaotic phenomena. ${ }^{4}$ These may be important effects in experiments and in technological devices, coherence having been suggested as a means of attaining practical microwave sources using arrays of coupled weak links, while chaos could be a threat to the proper functioning of the superconducting systems employed. The recent discovery of high- $T_{c}$ superconductivity which includes the Josephson effect, should spur new interest in such applications.

There are three mathematically simplest Josephson systems that have the requisite minimum threedimensional phase space 4 to display chaotic behavior: the single capacitive junction biased with dc currents and driven by an ac current, two resistively coupled noncapacitive ("overdamped") weak links dc biased and driven by an ac field, and three resistively coupled overdamped weak links de biased, but without a driving ac current.

The first system $^{5}$ has been examined for chaos extensively by means of numerical simulation as well as through the pendulum mechanical analogue. ${ }^{6}$ Furthermore, the existence of chaos has been demonstrated for the second. ${ }^{4}$

Results for the third system, ${ }^{7}$ the subject of this paper, have some bearing on use of arrays of weak links in technological applications including microwave sources and computer memories. Hadley and Beasley ${ }^{8}$ have published an interesting work on chaos and other phenomena in three coupled weak links, but with reactive coupling between junctions. This introduces derivatives higher than the first in the equations, which entails a phase space for their system of dimension considerably higher than 3 , thus disqualifying it from ranking among the mathematically simplest.
The equations of motion for a linear array of three resistively coupled Josephson weak links are ${ }^{3}$

$$
\begin{gathered}
\dot{\phi}_{1}=R_{1}\left[\left(I_{1}-I_{c_{1}} \sin \phi_{1}\right)+\alpha\left(I_{2}-I_{c_{2}}\right) \sin \phi_{2}\right], \\
\dot{\phi}_{2}=R_{2}\left[\left(I_{2}-I_{c_{2}} \sin \phi_{2}\right)+\alpha\left(I_{1}-I_{c_{1}}\right) \sin \phi_{1}\right. \\
\left.\quad+\alpha\left(I_{3}-I_{c_{3}} \sin \phi_{3}\right)\right], \\
\dot{\phi}_{3}=R_{3}\left[\left(I_{3}-I_{c_{3}} \sin \phi_{3}\right)+\alpha\left(I_{2}-I_{c_{2}}\right) \sin \phi_{2}\right] .
\end{gathered}
$$

$\phi_{i}, I_{c_{i}}, R_{i}$, and $I_{i}, i=1,2,3$, are the phase across the $i$ th link, the critical current and resistance of that link, and the $\mathrm{dc}$ bias current passing through it. Overdots refer to differentiation with respect to (normalized) time. ${ }^{9}$ The coupling represented by the parameter $\alpha$ may be produced by quasiparticle diffusion between junctions as well as by shunting the links via resistors. ${ }^{9}$ For simplicity the coupling is assumed equal for nearest neighbors, and negligible for non-nearest-neighbors.

The equations describe three phases $\phi_{1}, \phi_{2}$, and $\phi_{3}$ which can be taken to reside in a three-dimensional toroidal phase space, since the equations are periodic in each phase with period $2 \pi$. One expects three types of motion are possible for this sytem: coherent (periodic, or quasiperiodic with only two independent periods), quasiperiodic (i.e., triply periodic), and chaotic. The first is characterized by two or more of the average rates of winding (frequencies) of the three phases bearing a rational ratio to each other (voltage locking), $\left\langle\dot{\phi}_{i}\right\rangle:\left\langle\dot{\phi}_{j}\right\rangle=n: m$. The second is characterized by all three of these rates being in an irrational ratio to each other, with the motion regular in behavior and with discrete power spectra. The third has average rates of winding in irrational ratios to each other, but accompanied by apparent randomness of motion, noisy (continuous) spectra, and exponential divergence of neighboring trajectories (sensitivity to initial conditions).

In geometric terms coherence corresponds to trajectories which are closed curves in the three-torus, or closed curves in the two-dimensional subspace of the 
pair of locked junctions when only two links are locked. These trajectories are "attractors," in that any motion not starting on such a trajectory is attracted ever more closely to such sets as $t \rightarrow+\infty$. Attractors are concomitants of dissipation in the system with its associated phase-space volume shrinkage with time. Triply periodic motion fills the entire three-torus and is here associated with volume conservation in time. Contrasting sharply, chaotic motion usually results in a "strange attractor," that is, an attracting set having fractional dimension (fractal) and self-similar structure.

As mentioned above one can assign a dimensionality to a trajectory by means of a "correlation" function. ${ }^{10,11}$ This dimension calculation can be motivated by noting that a sampling of a $p$-dimensional object embedded in a higher-dimensional space will yield a number of points proportional to $r^{p}$ lying within a radius $r$ of a given point on it, at least for sufficiently small $r$. The correlation function is defined by

$$
C(r)=\frac{1}{N^{2}} \sum_{i=1}^{N} \sum_{\substack{j=1 \\(i \neq j)}}^{N} \theta\left(r-\left|\mathbf{X}_{i}-\mathbf{X}_{j}\right|\right)
$$

where $\theta(u)=1$ for $u>0, \theta(u)=0$ for $u \leq 0$, and $\left\{X_{i}\right\}$ is a set of $N$ sample points on the orbit. $N^{2} C(r)$ is the number of pairs, $\left(\mathbf{X}_{i}, \mathbf{X}_{j}\right)$, at a distance less than $r$. If for sufficiently small $r, C(r) \sim r^{\nu}$, then $v$ is referred to as the correlation dimension, a quantity which, while being an attribute similar to the Hausdorff (fractal) dimension, is somewhat more economically computed. The former dimension has been used recently to determine the possible existence of low-dimensional attractors in complex systems such as climate ${ }^{10,11}$ and the human brain. ${ }^{12}$

It is also of interest to discover the routes by which the onset of chaos occurs, the best known of which being the Feigenbaum scenario of a sequence of perioddoubling bifurcations. ${ }^{13}$ This seems to be the main route for the capacitive junction. Of more relevance in the present case is the Ruelle-Takens-Newhouse scenario, ${ }^{13,14}$ in which the very existence of a bifurcation from a solution with two to a solution with three independent frequencies, also harbors within it the potentiality of the otherwise unheralded onset of chaotic stochastic dynamics. More precisely, Ruelle and Takens ${ }^{14}$ show that in the neighborhood of a quasiperiodic solution involving at least three frequencies lie chaotic ("axiom $A$ ") attractors. This route was asserted as a counter to the suggestion first made by Landau in regard to turbulence $^{15}$ in which stochastic motion is presumed to occur only after an indefinitely large number of bifurcations; chaos being the limiting state of quasiperiodic motion with the number of independent frequencies tending to infinity.

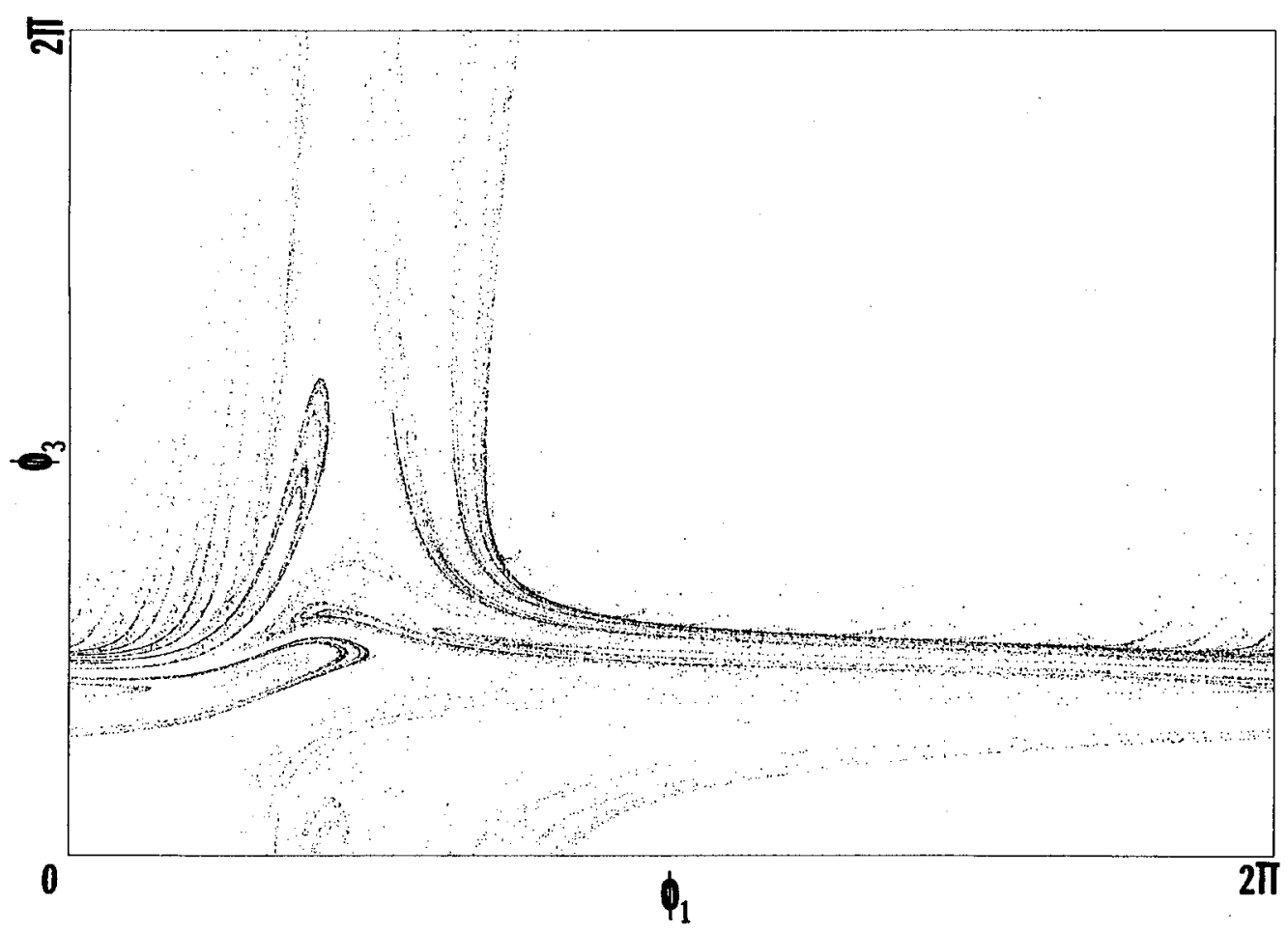

FIG. 1. A Poincaré plot using 20000 intersections of the trajectory with the two-torus $\phi_{2}=0(\bmod 2 \pi)$. Since we are in a toroidal phase space the opposite edges should be identified. The parameters are $I_{1}=2.0, I_{2}=2.309, I_{3}=2.0, I_{c_{1}}=0.9, I_{c_{2}}=0.9$, $I_{c_{3}}=1.2, R_{1}=1.1, R_{2}=1.0$, and $R_{3}=0.9$, with $\alpha=0.4$. The motion is chaotic with largest Lyapunov exponent of about 0.6 . This Poincare plot has correlation dimension of 1.67 ; hence the orbit is that of a strange attractor with correlation dimension of 2.67 . 


\section{RESULTS}

Equations (1) were solved in 17-decimal-digit arithmetic using a fourth-order variable-step-size RungeKutta method. The local error was controlled through the step size, ensuring reproducibility of the results. The largest Lyapunov exponent, the positive value of which being the definitive indication of chaos, was computed by the method of Bennetin et al. ${ }^{16}$
As well, the simulation monitored the relative rate of change of volume in phase space with time. If $W(t)$ is the volume of an "infinitesimal" element starting at $t=0$ with a volume $W_{0}$, and if the generic $n$-dimensional system satisfies $d \mathbf{x} / d t=\mathbf{f}(\mathbf{x})$, then it is easy to show

$$
\frac{1}{W} \frac{d W}{d t}=\sum_{i=1}^{n} \frac{\partial f_{i}}{\partial x_{i}},
$$
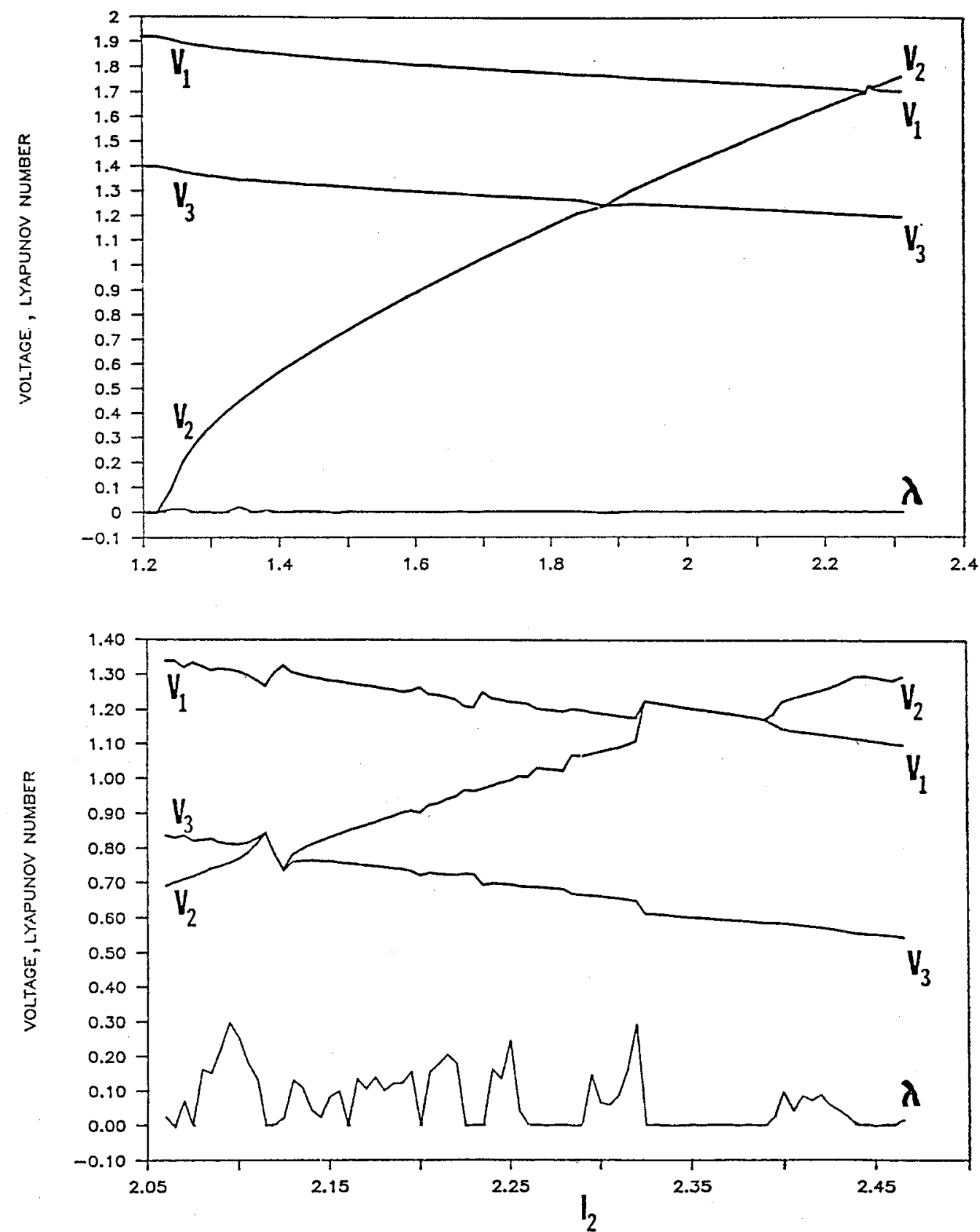

FIG. 2. $I-V$ curves for the three weak link system, with $I_{2}$ as the control parameter. The dc voltage $V_{i}$ is, in these units, $\left\langle\dot{\phi}_{i}\right\rangle$. $\alpha=0.3$ for the lower curve and $\alpha=0.1$ for the upper. All other parameters as for Fig. 1. The largest Lyapunov exponent, $\lambda$, is plotted as well (see text). 
which in our case from (1) becomes

$$
\frac{1}{W} \frac{d W}{d t}=-\sum_{i=1}^{3} R_{i} I_{c_{i}} \cos \phi_{i}
$$

In contrast to the single capacitive junction where the right-hand side of the corresponding equation is a negative constant, here the volume rate of change is variable. We find solutions to (1) such that the time average of $(1 / W)(d W / d t)$ is zero as well as negative. This indicates that some solutions are not attractors. When this ratio averages zero, the nature of the solution is quasiperiodic (with three independent frequencies) and vice versa. Unlike Hamiltonian systems, where $d W / d t \equiv 0$, yet chaos can nevertheless occur, here chaos is always associated with volume shrinkage with time. When $\langle(1 / W)(d W / d t)\rangle$ is less than 0 , the solutions either are phase locked (at least two of the three frequencies in rational relation to each other), or are chaotic with the attracting sets being fractals on the three-torus (see Fig. 1). It should be noted that in the case of weak coupling, with $\alpha$ as low as 0.1 , a "weaker" chaos, with low positive Lyapunov exponents, occurs. This happens only when one junction is operating at relatively low frequency compared to the other two. These solutions are still attractors but with relatively low rates of volume shrinkage. For higher frequencies at these low couplings the motion is nonchaotic. The triply periodic solutions would apparently fill the entire three-torus (technically dense in the three-torus), as indicated by Poincaré plots done with many thousands of points (not illustrated).
As the coupling is increased, triply periodic behavior disappears entirely leaving only chaos, except when locking of some frequencies (dc voltages) takes place or one of the junctions is passive, i.e., with dc voltage across it zero. Free-running solutions with their corresponding sets of three independent frequencies are apparently no longer possible. Figure 2 illustrates this by giving the $I-V$ curves with the largest Lyapunov exponents plotted on the same graph. For the smaller $\alpha$, quasiperiodic motion is the rule, except for very small intervals of locking, and a small region of "weak" chaos when the one junction is operating at low frequency. For the much higher $\alpha$ we see chaos prevails $(\lambda>0)$, except when there is coherence evidenced by the locking zones with their sharp edges.

One finds that chaos, totally absent at zero or very small coupling $(\alpha<0.1)$ and barely present at intermediate couplings $(0.1<\alpha<0.27)$, is generic when the coupling is sufficiently large. The exceptions occur when one of the junctions is passive (dc voltage is 0 ) or, the more interesting case, when two or more are locked. The value of $\alpha=0.27$ for this overall threshold for chaos will vary a small amount as other parameters are changed from the typical values chosen for Fig. 1.

As final corroboration of the determinations, the correlation dimensions of various solutions were computed. We find, as would be expected, that $v=3$ (to three decimal places) in the cases of triply periodic solutions, somewhat confirming the hypothesis that such trajectories fill the entire three-torus. For the chaotic solution
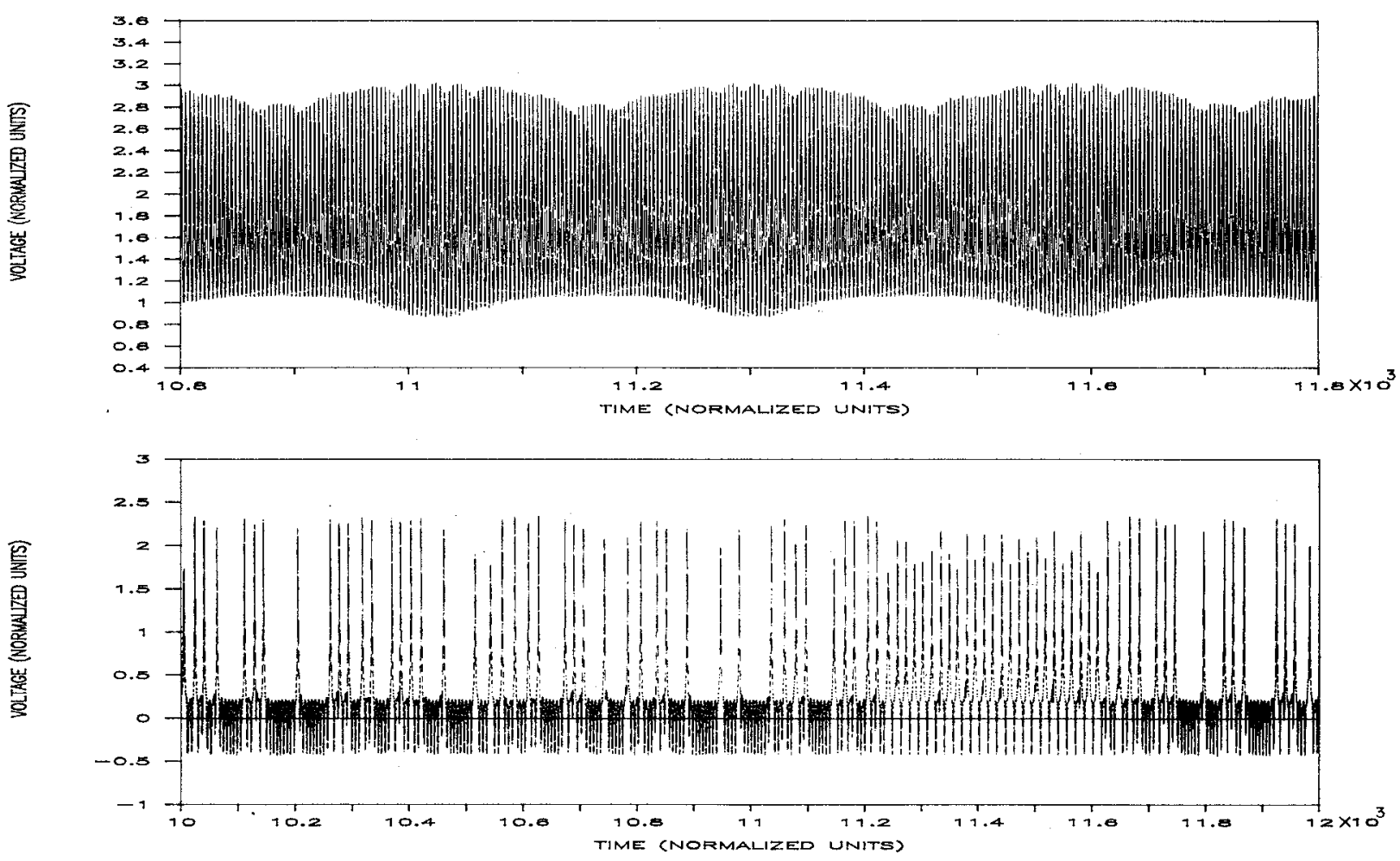

FIG. 3. Time traces of the voltage, $\dot{\phi}_{1}$, across link 1 for $\alpha=0.1$ and $0.4 . I_{2}=2.28$ for the former, while $I_{2}=2.309$ for the latter; all other parameters are as for Fig. 1. The lower trace is a voltage for a chaotic trajectory, while the upper one is that of a quasiperiodic trajectory. 
used in Fig. 1, this dimension was found to be 2.67 , indicating that the corresponding orbit is an attracting set not filling the three-torus, its fractional nature characterizing it as a "strange attractor," as opposed to a periodic attractor with dimension 1, or a partially coherent quasiperiodic attractor (two links locked, the third free running), which has a $v$ of 2 .

Careful study was made of the routes taken to chaos. Time traces of the voltages (e.g., Fig. 3) reveal that chaotic solutions are without long bursts of regular behavior, as might be expected from the MannevillePomeau intermittent chaos scenario. ${ }^{13}$ Exhaustive search does not find any period-doubling sequences near the onset of chaos, as the Feigenbaum scenario requires; hence we conclude that the aforementioned RuelleTakens-Newhouse (quasiperiodic) scenario is the only one. The observation of this route to chaos has, as well, been recently reported to occur in experiments on fluids. ${ }^{17,18}$

Parenthetically it should be finally remarked that despite allowing for a fairly long transient period during the simulations prior to commencing of Lyapunovexponent computations, one observes occasionally "transient chaos," a phenomenon reported in other studies. ${ }^{19}$ A solution may start out behaving chaotically, generating Poincaré plots very much like Fig. 1, only ultimately to fall into a coherent state, a periodic attractor. To ensure the reality of the observation of chaos, many trajec- tories were checked by allowing for extremely long transient periods in excess of $10^{6}$ time units.

\section{CONCLUSIONS}

We have shown by numerical simulation that sets of three strongly resistively coupled weak links will behave only coherently or chaotically under the effect solely of their mutual interaction. This would indicate that, experimentally, large arrays should be very "noisy" unless they are in some coherent state, at least when coupling between pairs is reasonably large, but nonetheless very much within the realm of experimental values. ${ }^{20,21}$ The onset of chaos in this system corroborates the contention that nonlinear systems with three or more frequencies are inherently unstable against stochastic motion. Further studies on much larger arrays are being carried out in order to determine whether thresholds for chaos drop with increasing number of links. In particular, the "weak" chaos observed at relatively low coupling might be more apparent in extended arrays. As well, larger arrays may go chaotic even when some subsets of links are mutually locked, providing only that three independent frequencies can be present.

\section{ACKNOWLEDGMENT}

This work was supported through grants from the Natural Science and Engineering Research Council of Canada.
1J. Bindslev Hansen and P. E. Lindelof, Rev. Mod. Phys. 56, 431 (1984).

${ }^{2}$ A. S. Deakin and M. A. H. Nerenberg, Phys. Rev. B 25, 1559 (1982).

${ }^{3}$ M. A. H. Nerenberg and J. A. Blackburn, Phys. Rev. B 23, 1149 (1981).

${ }^{4}$ M. A. H. Nerenberg, James A. Blackburn, and S. Vik, Phys. Rev. B 30, 5084 (1984).

${ }^{5}$ R. L. Kautz, IEEE Trans. Magn. MAG-19, 465 (1983).

${ }^{6}$ James A. Blackburn, Yang Zhou-Jing, S. Vik, H. J. T. Smith, and M. A. H. Nerenberg, Physica 26D, 385 (1987).

${ }^{7}$ Preliminary results on this system were first reported by the present authors at the MIDIT Workshop on Coherence and Chaos, Lyngby, Denmark, 1986 (unpublished).

${ }^{8}$ P. Hadley, and M. Beasley, Appl. Phys. Lett. 50, 621 (1987).

${ }^{9}$ D. W. Jillie, M. A. H. Nerenberg, and J. A. Blackburn, Phys. Rev. B 21, 125 (1980).

${ }^{10} \mathrm{C}$. Nicolis and G. Nicolis, Nature (London) 311, 529 (1984).

${ }^{11} \mathrm{C}$. Essex, T. Lookman, and M. A. H. Nerenberg, Nature (London) 326, 64 (1987).

${ }^{12}$ A. Babloyantz and A. Destexhe, Proc. Natl. Acad. Sci. U. S.
A. (Neurobiology) 83, 3513 (1987).

${ }^{13} \mathrm{D}$. Ruelle, in Turbulence and Predictability in Geophysical Fluid Dynamics and Climate Dynamics, edited by M. Ghil (North-Holland, Amsterdam, 1985).

${ }^{14}$ D. Ruelle and F. Takens, Commun. Math. Phys. 20, 167 (1971).

${ }^{15}$ A. S. Monin, Usp. Fiz. Nauk 125, 97 (1978) [Sov. Phys.Usp. 21, 429 (1978)].

${ }^{16} \mathrm{G}$. Bennetin, L. Galgani, and J. M. Strelcyn, Phys. Rev. A 14, 2338 (1976).

${ }^{17}$ J. Stavans, F. Heslot, and A. Libchaber, Phys. Rev. Lett. 55, 596 (1985).

${ }^{18} \mathrm{~K}$. R. Sreenivasan, in Frontiers in Fluid Mechanics, edited by S. H. Davis and J. L. Lumley (Springer-Verlag, Berlin, 1985), p. 41.

${ }^{19}$ J. P. Eckmann and D. Ruelle, Rev. Mod. Phys. 57, 617 (1985).

${ }^{20}$ D. W. Jillie, M. A. H. Nerenberg, and J. A. Blackburn, Phys. Rev. B 23, 1149 (1981).

${ }^{21}$ H. J. T. Smith (private communication). 